\title{
NEURAL NETWORK APPROXIMATION OF THE MASONRY FAILURE UNDER BIAXIAL COMPRESSIVE STRESS
}

\author{
Panagiotis G. Asteris ${ }^{1}$, Vagelis Plevris ${ }^{2}$ \\ ${ }^{1}$ Department of Civil \& Construction Engineering Educators \\ School of Pedagogical \& Technological Education \\ Heraklion, GR 141 21, Athens, Greece \\ ${ }^{2}$ Department of Civil \& Structural Engineering Educators \\ School of Pedagogical \& Technological Education \\ Heraklion, GR 141 21, Athens, Greece \\ e-mail: asteris@aspete.gr, vplevris@gmail.com
}

Keywords: Masonry, anisotropy, failure criterion, failure surface, biaxial stress, Neural Network, NN, approximation.

\begin{abstract}
Masonry is a material that exhibits distinct directional properties because the mortar joints act as planes of weakness. To define failure under biaxial stress, a threedimensional surface in terms of the two normal stresses and shear stress, or the two principal stresses and their orientation to the bed joints, is required.

Researchers have long been aware of the significance of the bed joint angle to the applied load and many experimental tests have been carried out on brick masonry discs to produce indirect tensile stresses on joints inclined at various angles to the vertical compressive load. The highest strength of the masonry has been observed for the cases when the compressive load was perpendicular to the bed joints or when the principal tensile stress at the center of the disc was parallel to the bed joints. In this case failure occurred through bricks and perpendicular joints. The lowest strength has been observed when the compressive load was parallel to the bed joints or when the principal tensile stress at the center of the disc was perpendicular to the bed joints. In this case failure occurred along the interface of brick and mortar joint.

In the present study, the preliminary results of an ongoing research on the failure of brittle anisotropic materials are presented. In particular, Neural Networks (NNs) are used in order to approximate the experimental failure curves of a brittle anisotropic material such as masonry, that has been investigated in depth by Page [1]. For each angle $\theta\left(0^{\circ}, 22.5^{\circ}, 45^{\circ}\right), a$ Neural Network is trained with the experimental data of Page as inputs. Then the NN is asked to produce the whole failure curve for each angle as its output, filling also the gaps between the experimental points with appropriate approximations. The results show the great potential of using NN for the approximation of the masonry failure under biaxial compressive stress.
\end{abstract}




\section{INTRODUCTION}

Despite the fact that masonry is one of the oldest structural materials and, actually, the main element in monumental structures such as churches, castles, mosques etc., our knowledge regarding its mechanical behavior is not as thorough as it should be and many aspects of its behavior remain to be investigated. One reason for this lack of extensive studies is the highly anisotropic brittle nature of masonry, which makes complicated, difficult and expensive, the realization of reliable experimental tests under conditions of biaxial stress, and, even more, under conditions of biaxial tension or heterosemous stress. Taking into account the numerous uncertainties of the problem, a computational model, describing the masonry failure surface in a simple manner should be an efficient tool for the investigation of the behavior of masonry structures. Many analytical criteria for masonry structures have been already proposed [2-7]. Experimental investigations can also be considered as an important support to the aforementioned efforts $[1,8,9,10]$.

In the present study, we use the experimental data reported by Page [1], referring to a total of 102 panels, that have been already used by many other researchers $[2,3,11]$. Ratios of vertical compressive stress $\sigma_{1}$ to horizontal compressive stress $\sigma_{2}$ of $\infty$ (uniaxial $\sigma_{1}$ ), 10, 4, 2 and 1 have been used in conjunction with a bed joint angle $\theta$ with respect to the $\sigma_{1}$, in directions of $0^{\circ}, 22.5^{\circ}, 45^{\circ}, 67.5^{\circ}$ and $90^{\circ}$. A minimum of four tests were performed for each combination of $\sigma_{1}, \sigma_{2}$ and $\theta$. The aim of the present paper is to introduce an anisotropic (orthotropic) Neural Network - generated failure surface under biaxial stress for masonry.

\section{LITERATURE REVIEW}

Masonry exhibits distinct directional properties due to the influence of the mortar joints. Depending upon the orientation of the joints to the stress directions, failure can occur in the joints alone or simultaneously in the joints and the blocks. The failure of masonry under uniaxial and biaxial stress state has been studied experimentally in the past by many researchers but to the authors' knowledge there has not been any attempt to apply a Neural Network for such a prediction.

Only a few studies incorporate the use of Neural Networks (NNs) for the approximation of masonry behavior in general. Zhang et al. in [12] apply artificial intelligent techniques for directly predicting the cracking patterns of masonry wallets, subjected to vertical loading. The von Neumann neighborhood model and the Moore neighborhood model of cellular automata (CA) are used to establish the CA numerical model for masonry wallets. Techniques for the analysis of wallets whose bed courses have different angles with the horizontal bottom edges are also introduced. Two criteria are used to match zone similarity between a "base wallet" and any new "unseen" wallets. This zone similarity information is used to predict the cracks in unseen wallets. A back-propagation neural network is also used for predicting the cracking pattern of a wallet based on the CA model of the wallet and some data of recorded cracking at zones.

El-Shafie et al. [13] propose a model based on radial basis function neural networks (RBFNN) for predicting creep in concrete and masonry structures and is compared to a multilayer perceptron neural network (MLPNN). Accurate prediction of creep is achieved due to the simple architecture and fast training procedure of RBFNN model especially when compared to MLPNN model. The RBFNN model shows good agreement with experimental creep data from brickwork assemblages.

Zhou et al. [14] propose an artificial intelligent technique for predicting the failure/cracking loads of laterally loaded masonry wall panels based on their corresponding failure/cracking patterns derived from the laboratory experiments. The numerical modes of 
failure/cracking patterns of experimental wall panels and the corresponding normalized failure/cracking loads are used as the input and output for the NN training. Three types of NN models for predicting the failure/cracking load of the unseen wall panel are achieved by repeatedly training and adjusting to optimize its parameters.

Mathew et al. [15] propose the use of NN for solving complex nonlinear problems for the analysis of masonry panels under biaxial bending. A Neural Network, trained with the use of a set of data, which is representative of the problem domain, is shown to be successful in solving new problems with reasonable accuracy. The experimental results obtained from the testing of panels are analyzed, and the method that gives good correlation between the theoretical prediction and the experimental result is recommended for other panels of similar properties and boundary conditions. An artificial intelligence based technology, the casebased reasoning (CBR), has been used to solve new problems by adapting solutions to similar problems solved in the past, which are stored in the case library. A hybrid system is described that utilizes the capabilities of both ANNs and CBR.

\section{EXPERIMENTAL DATA}

The experimental data of Page [1] have been used as inputs for the NN model in the present study. The figures below show the original data together with the mean curves for each bed joint angle that attempt to fit these data.

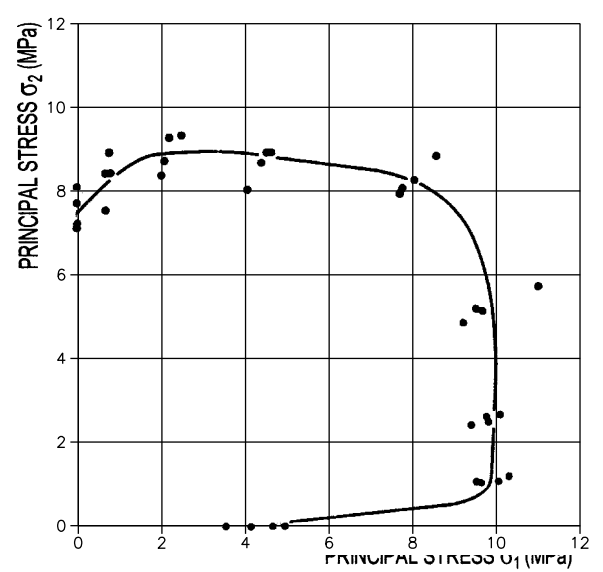

(a)

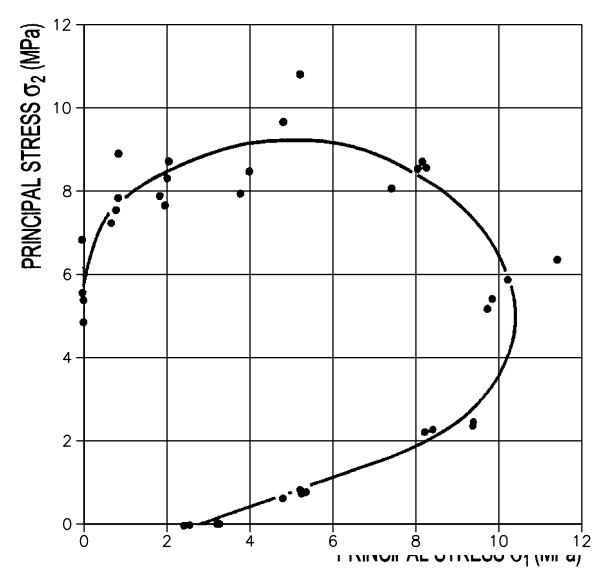

(b)

Figure 1: Failure of Brickwork under Biaxial Compression, (a) $\theta=0^{\circ}$, (b) $\theta=22.5^{\circ}$ [1].

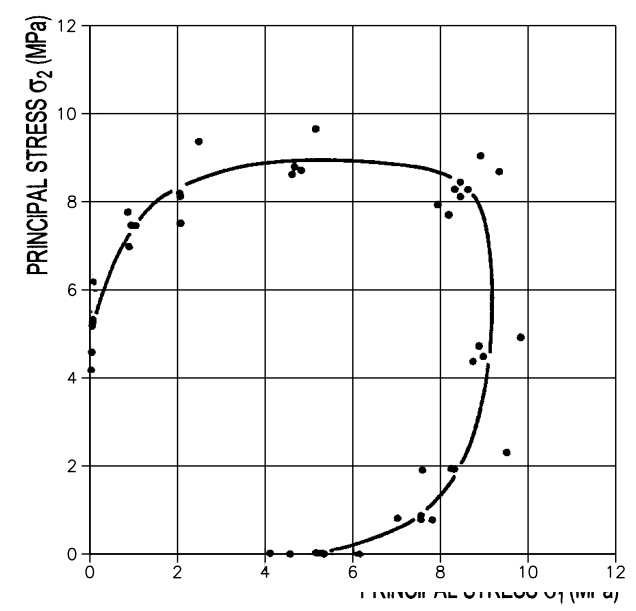

Figure 2: Failure of Brickwork under Biaxial Compression, $\theta=45^{\circ}[1]$. 
It should be noted that the data for $\theta=45^{\circ}$ are symmetric to the $45^{\circ}$ line $\left(\sigma_{1}=\sigma_{2}\right)$, due to the nature of the loading, while the cases $\theta=67.5^{\circ}$ and $\theta=90^{\circ}$ are equivalent to the cases $\theta=22.5^{\circ}$ and $\theta=0^{\circ}$, respectively and will not be examined separately. The table below shows the analytical data for the $\theta=0^{\circ}$ case.

\begin{tabular}{|c|c|c|c|c|c|c|c|}
\hline$\sigma_{\mathrm{I}}(\mathrm{MPa})$ & $\sigma_{\|}(\mathrm{MPa})$ & $\sigma_{\mathrm{I}} / \mathbf{f}_{\mathrm{wc}}$ & $\sigma_{\mathrm{H}} / \mathbf{f}_{\mathrm{wc}}$ & $\begin{array}{c}\text { Average } \\
\sigma_{1} / f_{w c}\end{array}$ & $\begin{array}{c}\text { Average } \\
\sigma_{\| /} / \mathbf{f}_{\mathrm{wc}}\end{array}$ & $\begin{array}{c}\text { Angle } \varphi= \\
\operatorname{Atan}\left(\sigma_{\|} / \sigma_{\mathrm{I}}\right)\end{array}$ & Radius $r$ \\
\hline 3.53 & 0.00 & 0.47 & 0.00 & & & & \\
\hline 4.14 & 0.00 & 0.55 & 0.00 & & & & \\
\hline 4.66 & 0.00 & 0.62 & 0.00 & & & & \\
\hline 4.96 & 0.00 & 0.66 & 0.00 & 0.57 & 0.00 & 0.0000 & 0.5717 \\
\hline 9.59 & 0.85 & 1.27 & 0.11 & & & & \\
\hline 9.70 & 0.87 & 1.28 & 0.12 & & & & \\
\hline 10.11 & 0.90 & 1.34 & 0.12 & & & & \\
\hline 10.37 & 1.01 & 1.37 & 0.13 & 1.32 & 0.12 & 0.0909 & 1.3210 \\
\hline 9.46 & 2.26 & 1.25 & 0.30 & & & & \\
\hline 9.85 & 2.43 & 1.30 & 0.32 & & & & \\
\hline 9.92 & 2.30 & 1.31 & 0.30 & & & & \\
\hline 10.15 & 2.45 & 1.34 & 0.32 & 1.30 & 0.31 & 0.2353 & 1.3398 \\
\hline 9.33 & 4.67 & 1.23 & 0.62 & & & & \\
\hline 9.62 & 5.01 & 1.27 & 0.66 & & & & \\
\hline 9.74 & 4.95 & 1.29 & 0.65 & & & & \\
\hline 11.15 & 5.48 & 1.48 & 0.72 & 1.32 & 0.66 & 0.4672 & 1.4761 \\
\hline 7.84 & 7.76 & 1.04 & 1.03 & & & & \\
\hline 7.89 & 7.91 & 1.04 & 1.05 & & & & \\
\hline 8.20 & 8.10 & 1.09 & 1.07 & & & & \\
\hline 8.72 & 8.67 & 1.15 & 1.15 & 1.08 & 1.07 & 0.7820 & 1.5222 \\
\hline 4.16 & 7.95 & 0.55 & 1.05 & & & & \\
\hline 4.55 & 8.57 & 0.60 & 1.13 & & & & \\
\hline 4.66 & 8.84 & 0.62 & 1.17 & & & & \\
\hline 4.74 & 8.81 & 0.63 & 1.17 & 0.60 & 1.13 & 1.0836 & 1.2792 \\
\hline 2.12 & 8.34 & 0.28 & 1.10 & & & & \\
\hline 2.21 & 8.67 & 0.29 & 1.15 & & & & \\
\hline 2.32 & 9.21 & 0.31 & 1.22 & & & & \\
\hline 2.63 & 9.32 & 0.35 & 1.23 & 0.31 & 1.18 & 1.3156 & 1.2148 \\
\hline 0.76 & 7.55 & 0.10 & 1.00 & & & & \\
\hline 0.76 & 8.42 & 0.10 & 1.11 & & & & \\
\hline 0.87 & 8.42 & 0.11 & 1.11 & & & & \\
\hline 0.87 & 8.93 & 0.11 & 1.18 & 0.11 & 1.10 & 1.4737 & 1.1074 \\
\hline 0.00 & 7.15 & 0.00 & 0.95 & & & & \\
\hline 0.00 & 7.27 & 0.00 & 0.96 & & & & \\
\hline 0.00 & 7.69 & 0.00 & 1.02 & & & & \\
\hline 0.00 & 8.12 & 0.00 & 1.07 & 0.00 & 1.00 & 1.5708 & 1.0000 \\
\hline
\end{tabular}

Table 1. Failure of Brickwork under Biaxial Compression, $\theta=0^{\circ}[1]$ and relevant calculations.

The first two columns of the table contain the original experimental data, namely the failure principal stresses $\sigma_{\mathrm{I}}$ and $\sigma_{\mathrm{II}}$ in MPa. For every loading case, four tests have been conducted. The next two columns contain the same data in a dimensionless form where the stresses have been divided with the stress $f_{\mathrm{wc}}$ which is the masonry strength for the case $\sigma_{\mathrm{I}}=0$ (last four rows of the table). The value of $f_{\mathrm{wc}}$ has been calculated as the average of the four values (high- 
lighted in bold in the above table) as $7.56 \mathrm{MPa}$. The next two columns are the averages of the four tests for each loading case.

For the data to be suitable for the Neural Network training, a conversion to polar coordinates $(r, \varphi)$ has been taken place, where the radius $r$ is given by

$$
r=\sqrt{\sigma_{I, \text { aver }}^{2}+\sigma_{I I, \text { aver }}^{2}}
$$

where $\sigma_{\mathrm{I}, \text { aver }}$ and $\sigma_{\mathrm{II}, \mathrm{aver}}$ are the average stresses for each loading case (columns 5, 6). The polar angle $\varphi$ (column 7) is given by

$$
\varphi=\operatorname{Arctan}\left(\frac{\sigma_{I I}}{\sigma_{I}}\right)
$$

The polar radius $r$ is given in the last column (column 8) of the above table. For each angle $\theta$ case $\left(\theta=0^{\circ}, 22.5^{\circ}, 45^{\circ}\right)$, a Neural Network is trained with the angle $\varphi$ as its input and the radius $r$ as its output. The figure below shows the data of the above table, where also the average values (used for the NN training) have been highlighted with red rectangles.

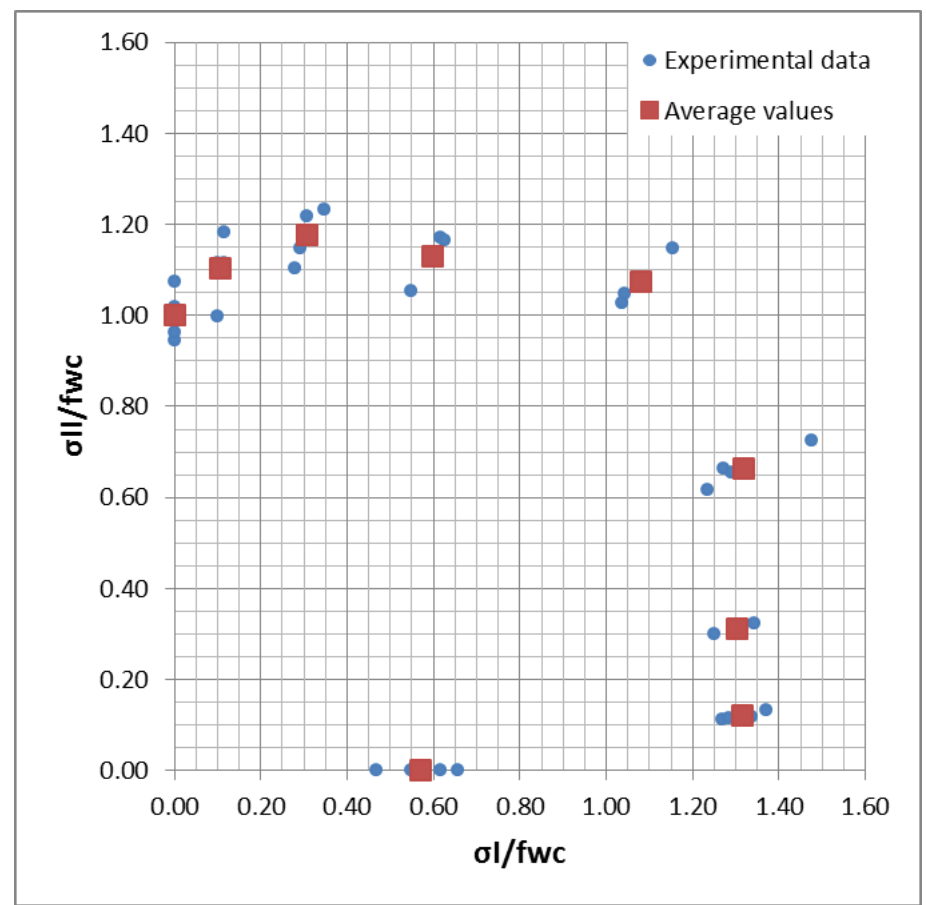

Figure 3: Normalized experimental data and average values for the $\theta=0^{\circ}$ case.

The tables and figures below show the corresponding data for the other two cases $\left(\theta=22.5^{\circ}\right.$ and $\theta=45^{\circ}$ ). 
Panagiotis G.Asteris and Vagelis Plevris

\begin{tabular}{|c|c|c|c|c|c|c|c|}
\hline$\sigma_{\mathrm{I}}(\mathrm{MPa})$ & $\sigma_{\|}(\mathrm{MPa})$ & $\sigma_{1} / f_{w c}$ & $\sigma_{\|} / \mathbf{f}_{\mathrm{wc}}$ & $\begin{array}{c}\text { Average } \\
\sigma_{\mathrm{l}} / \mathbf{f}_{\mathrm{wc}}\end{array}$ & $\begin{array}{c}\text { Average } \\
\sigma_{\|} / \mathbf{f}_{\mathrm{wc}}\end{array}$ & $\begin{array}{c}\text { Angle } \varphi= \\
\operatorname{Atan}\left(\sigma_{\|} / \sigma_{l}\right)\end{array}$ & Radius \\
\hline 2.37 & 0.00 & 0.31 & 0.00 & & & & \\
\hline 2.50 & 0.00 & 0.33 & 0.00 & & & & \\
\hline 3.19 & 0.00 & 0.42 & 0.00 & & & & \\
\hline 3.28 & 0.00 & 0.43 & 0.00 & 0.38 & 0.00 & 0.0000 & 0.3752 \\
\hline 4.79 & 0.60 & 0.63 & 0.08 & & & & \\
\hline 5.24 & 0.78 & 0.69 & 0.10 & & & & \\
\hline 5.22 & 0.71 & 0.69 & 0.09 & & & & \\
\hline 5.38 & 0.71 & 0.71 & 0.09 & 0.68 & 0.09 & 0.1352 & 0.6890 \\
\hline 8.26 & 2.08 & 1.09 & 0.27 & & & & \\
\hline 8.47 & 2.12 & 1.12 & 0.28 & & & & \\
\hline 9.44 & 2.18 & 1.25 & 0.29 & & & & \\
\hline 9.47 & 2.27 & 1.25 & 0.30 & 1.18 & 0.29 & 0.2382 & 1.2132 \\
\hline 9.84 & 4.96 & 1.30 & 0.66 & & & & \\
\hline 9.95 & 5.22 & 1.32 & 0.69 & & & & \\
\hline 10.33 & 5.65 & 1.37 & 0.75 & & & & \\
\hline 11.56 & 6.10 & 1.53 & 0.81 & 1.38 & 0.73 & 0.4843 & 1.5581 \\
\hline 7.57 & 7.89 & 1.00 & 1.04 & & & & \\
\hline 8.22 & 8.31 & 1.09 & 1.10 & & & & \\
\hline 8.35 & 8.53 & 1.10 & 1.13 & & & & \\
\hline 8.46 & 8.35 & 1.12 & 1.10 & 1.08 & 1.09 & 0.7927 & 1.5359 \\
\hline 3.91 & 7.83 & 0.52 & 1.04 & & & & \\
\hline 4.12 & 8.37 & 0.54 & 1.11 & & & & \\
\hline 4.98 & 9.54 & 0.66 & 1.26 & & & & \\
\hline 5.43 & 10.65 & 0.72 & 1.41 & 0.61 & 1.20 & 1.1018 & 1.3493 \\
\hline 2.06 & 7.59 & 0.27 & 1.00 & & & & \\
\hline 1.95 & 7.84 & 0.26 & 1.04 & & & & \\
\hline 2.15 & 8.27 & 0.28 & 1.09 & & & & \\
\hline 2.20 & 8.65 & 0.29 & 1.14 & 0.28 & 1.07 & 1.3178 & 1.1053 \\
\hline 0.76 & 7.23 & 0.10 & 0.96 & & & & \\
\hline 0.89 & 7.53 & 0.12 & 1.00 & & & & \\
\hline 0.93 & 7.80 & 0.12 & 1.03 & & & & \\
\hline 0.98 & 8.89 & 0.13 & 1.18 & 0.12 & 1.04 & 1.4580 & 1.0467 \\
\hline 0.00 & 4.87 & 0.00 & 0.64 & & & & \\
\hline 0.00 & 5.39 & 0.00 & 0.71 & & & & \\
\hline 0.00 & 5.59 & 0.00 & 0.74 & & & & \\
\hline 0.00 & 6.85 & 0.00 & 0.91 & 0.00 & 0.75 & 1.5708 & 0.7508 \\
\hline
\end{tabular}

Table 2. Failure of Brickwork under Biaxial Compression, $\theta=22.5^{\circ}$ [1] and relevant calculations. 
Panagiotis G.Asteris and Vagelis Plevris

\begin{tabular}{|c|c|c|c|c|c|c|c|}
\hline $\begin{array}{c}\sigma_{\mathrm{I}} \\
(\mathrm{MPa})\end{array}$ & $\sigma_{\|}(\mathrm{MPa})$ & $\sigma_{\mathrm{I}} / \mathbf{f}_{\mathrm{wc}}$ & $\sigma_{\|} / \mathbf{f}_{\mathrm{wc}}$ & $\begin{array}{c}\text { Average } \\
\sigma_{I} / f_{w c}\end{array}$ & $\begin{array}{c}\text { Average } \\
\sigma_{\|} / \mathbf{f}_{\mathrm{wc}}\end{array}$ & $\begin{array}{c}\text { Angle } \varphi= \\
\operatorname{Atan}\left(\sigma_{\| I} / \sigma_{I}\right)\end{array}$ & Radius $r$ \\
\hline 4.09 & 0.00 & 0.54 & 0.00 & & & & \\
\hline 4.56 & 0.00 & 0.60 & 0.00 & & & & \\
\hline 5.14 & 0.00 & 0.68 & 0.00 & & & & \\
\hline 5.29 & 0.00 & 0.70 & 0.00 & & & & \\
\hline 6.11 & 0.00 & 0.81 & 0.00 & 0.67 & 0.00 & 0.0000 & 0.6666 \\
\hline 6.99 & 0.77 & 0.93 & 0.10 & & & & \\
\hline 7.52 & 0.72 & 0.99 & 0.10 & & & & \\
\hline 7.52 & 0.80 & 0.99 & 0.11 & & & & \\
\hline 7.76 & 0.74 & 1.03 & 0.10 & 0.99 & 0.10 & 0.1012 & 0.9905 \\
\hline 7.54 & 1.88 & 1.00 & 0.25 & & & & \\
\hline 8.19 & 1.88 & 1.08 & 0.25 & & & & \\
\hline 8.27 & 1.85 & 1.09 & 0.24 & & & & \\
\hline 9.50 & 2.24 & 1.26 & 0.30 & 1.11 & 0.26 & 0.2301 & 1.1379 \\
\hline 8.71 & 4.32 & 1.15 & 0.57 & & & & \\
\hline 8.83 & 4.70 & 1.17 & 0.62 & & & & \\
\hline 8.94 & 4.45 & 1.18 & 0.59 & & & & \\
\hline 9.80 & 4.86 & 1.30 & 0.64 & 1.20 & 0.61 & 0.4677 & 1.3446 \\
\hline 8.14 & 7.64 & 1.08 & 1.01 & & & & \\
\hline 8.41 & 8.05 & 1.11 & 1.07 & & & & \\
\hline 8.59 & 8.22 & 1.14 & 1.09 & & & & \\
\hline 9.32 & 8.61 & 1.23 & 1.14 & 1.14 & 1.08 & 0.7565 & 1.5675 \\
\hline 7.64 & 8.14 & 1.01 & 1.08 & & & & \\
\hline 8.05 & 8.41 & 1.07 & 1.11 & & & & \\
\hline 8.22 & 8.59 & 1.09 & 1.14 & & & & \\
\hline 8.61 & 9.32 & 1.14 & 1.23 & 1.08 & 1.14 & 0.8143 & 1.5675 \\
\hline 4.32 & 8.71 & 0.57 & 1.15 & & & & \\
\hline 4.70 & 8.83 & 0.62 & 1.17 & & & & \\
\hline 4.45 & 8.94 & 0.59 & 1.18 & & & & \\
\hline 4.86 & 9.80 & 0.64 & 1.30 & 0.61 & 1.20 & 1.1031 & 1.3446 \\
\hline 1.88 & 7.54 & 0.25 & 1.00 & & & & \\
\hline 1.88 & 8.19 & 0.25 & 1.08 & & & & \\
\hline 1.85 & 8.27 & 0.24 & 1.09 & & & & \\
\hline 2.24 & 9.50 & 0.30 & 1.26 & 0.26 & 1.11 & 1.3407 & 1.1379 \\
\hline 0.77 & 6.99 & 0.10 & 0.93 & & & & \\
\hline 0.72 & 7.52 & 0.10 & 0.99 & & & & \\
\hline 0.80 & 7.52 & 0.11 & 0.99 & & & & \\
\hline 0.74 & 7.76 & 0.10 & 1.03 & 0.10 & 0.99 & 1.4696 & 0.9905 \\
\hline 0.00 & 4.09 & 0.00 & 0.54 & & & & \\
\hline 0.00 & 4.56 & 0.00 & 0.60 & & & & \\
\hline 0.00 & 5.14 & 0.00 & 0.68 & & & & \\
\hline 0.00 & 5.29 & 0.00 & 0.70 & & & & \\
\hline 0.00 & 6.11 & 0.00 & 0.81 & 0.00 & 0.67 & 1.5708 & 0.6666 \\
\hline
\end{tabular}

Table 3. Failure of Brickwork under Biaxial Compression, $\theta=45^{\circ}[1]$ and relevant calculations. 


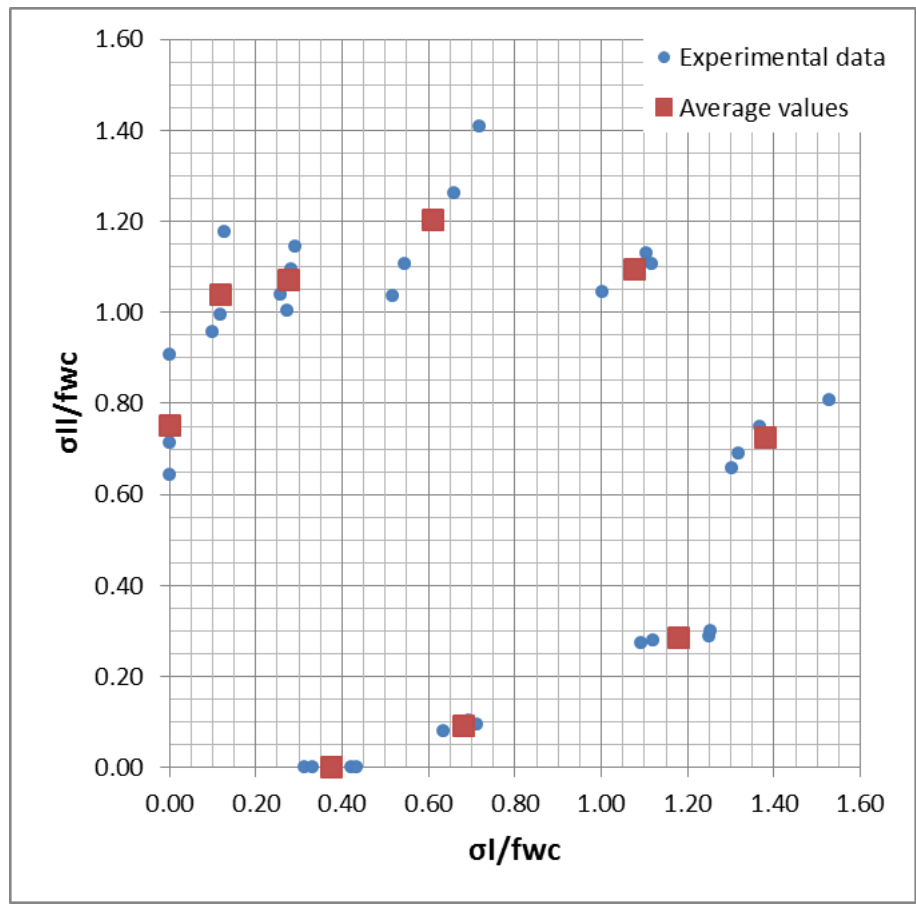

Figure 4: Normalized experimental data and average values for the $\theta=22.5^{\circ}$ case.

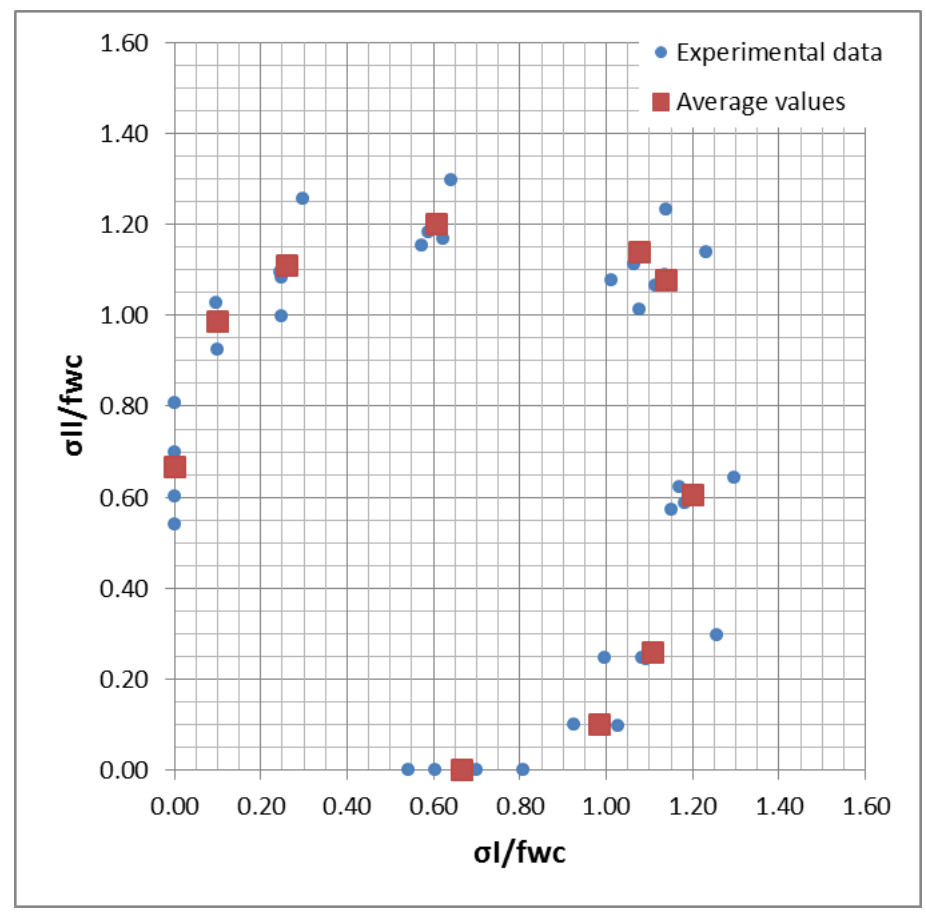

Figure 5: Normalized experimental data and average values for the $\theta=45^{\circ}$ case.

\section{ARTIFICIAL NEURAL NETWORKS}

The development of artificial neural networks was initially inspired and motivated by insights into how biological brains - and in particular mammalian brains - function. It was found that mammalian brains learn as connections between neurons are strengthened - the result of electrochemical processes triggered by external or internal stimuli (experiences). As 
in biological systems, learning involves adjustments to the synaptic connections that exist between the neurons. NNs, like human beings, learn by example.

Although parallels with biological systems are often described, there is still so little known (even at the lowest cell level) about biological systems, that the models that are being used for artificial neural systems seem to introduce an oversimplification of the 'biological' models. The real, biological nervous system is highly complex and includes some features that may seem superfluous based on an understanding of artificial networks.

In the first work on the processing of neural networks [16], it was shown theoretically that networks of artificial neurons could implement logical, arithmetic, and symbolic functions. Simplified models of biological neurons were set up, now usually called perceptrons or artificial neurons. These simple models accounted for neural summation, i.e. potentials at the postsynaptic membrane would sum in the cell body. Later models also provided for excitatory and inhibitory synaptic transmission.

Artificial Neural Networks are made up of fully or partially interconnecting artificial neurons (programming constructs that mimic the properties of biological neurons). Artificial neural networks may either be used to gain an understanding of biological neural networks, or for solving artificial intelligence problems without necessarily creating a model of a real biological system. Artificial Intelligence (AI) and cognitive modeling try to simulate some properties of neural networks. While similar in their techniques, AI has the aim of solving particular tasks, while cognitive modeling aims to build mathematical models of biological neural systems. In the AI field, artificial neural networks have been trained to perform complex functions in various scientific fields and have been applied successfully to identification, classification, simulation, inverse simulation, speech recognition, pattern recognition, image analysis and adaptive control, and also in order to construct software agents (in computer games) or autonomous robots.

\section{NEURAL NETWORKS ARCHITECTURE}

In the present study, we use a Back-Propagation Neural Network (BPNN). Here, the output values are compared with the correct answer to compute the value of a predefined errorfunction. By various techniques, the error is then fed back through the network. Using this information, the algorithm adjusts the weights of each connection in order to reduce the value of the error function by some small amount. After repeating this process for a sufficiently large number of training cycles, the network will usually converge to some state where the error of the calculations is small. In this case, one would say that the network has learned a certain target function. As the algorithm's name implies, the errors propagate backwards from the output nodes to the inner nodes. So technically speaking, back-propagation is used to calculate the gradient of the error of the network with respect to the network's modifiable weights. To adjust weights properly, one applies a general method for non-linear optimization that is called gradient descent. In order to minimize the error, the derivative of the error function with respect to the network weights is calculated, and the weights are then changed such that the error decreases (thus going downhill on the surface of the error function). For this reason, back-propagation can only be applied on networks with differentiable activation functions. Back-propagation usually allows quick convergence on satisfactory local minima for error in the kind of networks to which it is suited.

A BPNN is a feed-forward, multilayer network of standard structure, i.e. neurons are not connected in the layer but they join the layer neuron with all the neurons of previous and subsequent layers, respectively. A BPNN has a standard structure that can be written in short as

$$
N-H_{1}-H_{2}-\ldots-H_{N L-1}-M
$$


where $N$ is the number of inputs, $H_{l}$ is the number of neurons in the $l$-th hidden layer, $N L$ is the number of layers (including the output layer) and $M$ is the number of output neurons. Figure 6 depicts an example of a BPNN composed of an input layer with 4 neurons, two hidden layers with 3 neurons each and an output layer with 2 neurons, i.e. a 4-3-3-2 BPNN.

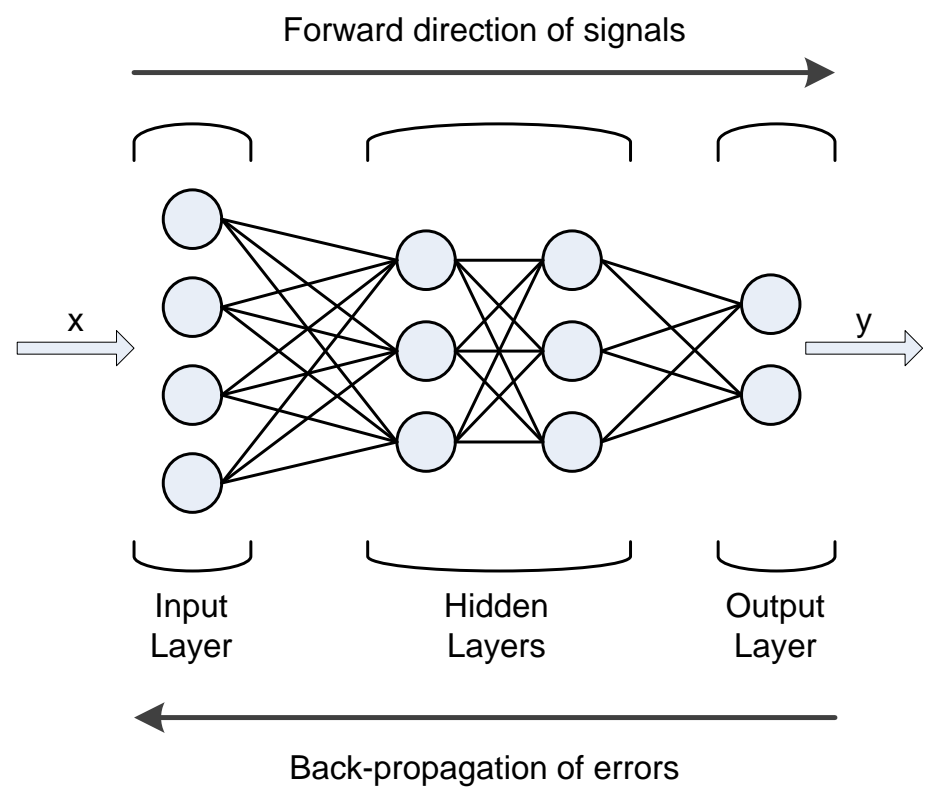

Figure 6: A three-layer 4-3-3-2 BPNN (input not counted as a layer).

In the present study, we use a Back-Propagation Neural Network with two hidden layers, one input layer and one output layer. The input layer has one node (neuron) which corresponds to the angle $\varphi$, while the output layer has also one node which corresponds to the radius $r$. The two hidden layers have 8 nodes each, ending in a 1-8-8-1 BPNN architecture, for the first two cases $\left(\theta=0^{\circ}\right.$ and $\left.\theta=22.5^{\circ}\right)$. For the third case $\left(\theta=45^{\circ}\right)$ the two hidden layers have 12 nodes each, ending in a 1-12-12-1 BPNN architecture. The input and output values are normalized before the NN training and the inverse normalization is done in order to take the NN results for other data afterwards.

A single node (neuron) of a hidden layer, with a single $R$-element input vector is shown below.

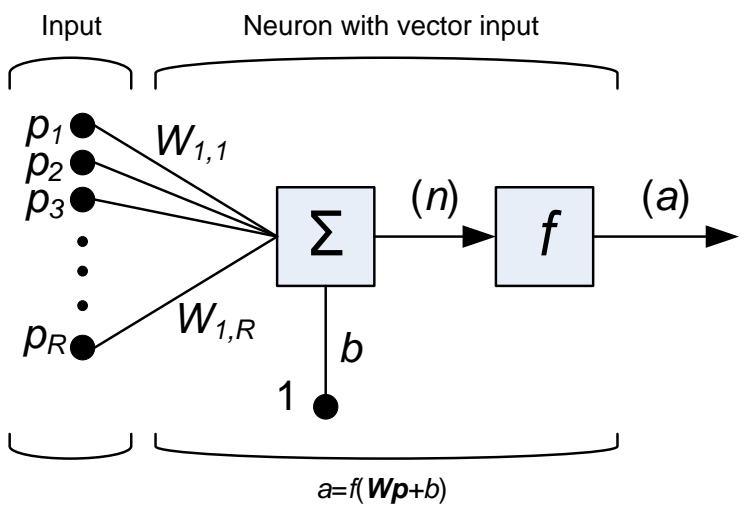

Figure 7: A neuron with a single R-element input vector. 
For each node, the individual element inputs $p_{1}, \ldots, p_{R}$ are multiplied by weights $w_{1,1}, \ldots$, $w_{1, \mathrm{R}}$ and the weighted values are fed to the summing junction. The sum is $\boldsymbol{W} \cdot \boldsymbol{p}$, the dot product of the (single row) matrix $\boldsymbol{W}=\left[w_{1,1}, \ldots, w_{1, R}\right]$ and the vector $\boldsymbol{p}=\left[p_{1}, \ldots, p_{\mathrm{R}}\right]^{\mathrm{T}}$. The neuron has a bias $b$, which is summed with the weighted inputs to form the net input $n$ which is the argument of the transfer function $f$ :

$$
n=w_{1,1} p_{1}+w_{1,2} p_{2}+\ldots+w_{1, R} p_{R}+b=\boldsymbol{W} \cdot \boldsymbol{p}+b
$$

In the above case, $\boldsymbol{p}$ is a column vector $(R \times 1), \boldsymbol{W}$ is a row vector $(1 \times R)$ and $b$ is a scalar.

\subsection{Transfer function}

The transfer function used is the hyperbolic tangent function, the same for all the hidden and the input layer, while the transfer function for the output layer is a linear function. The output of the hyperbolic tangent function and its derivative are given by

$$
\begin{gathered}
a=f(n)=\frac{e^{2 n}-1}{e^{2 n}+1}=i \cdot \tan (i x) \\
f^{\prime}(n)=4 \frac{e^{2 n}}{\left(e^{2 n}+1\right)^{2}}=1-a^{2}
\end{gathered}
$$

This function yields output values in the interval [-1,1], while its derivative yields output values in the interval $[0,1]$. The graphical representations of the hyperbolic tangent function and its derivative are shown in the figure below.

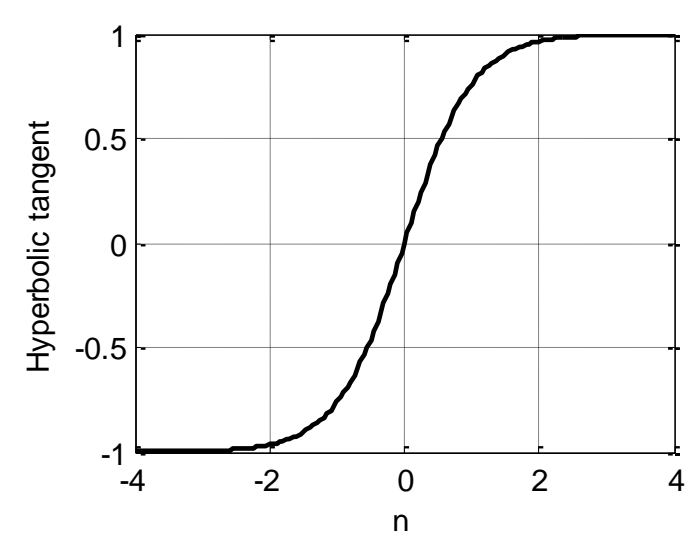

(a)

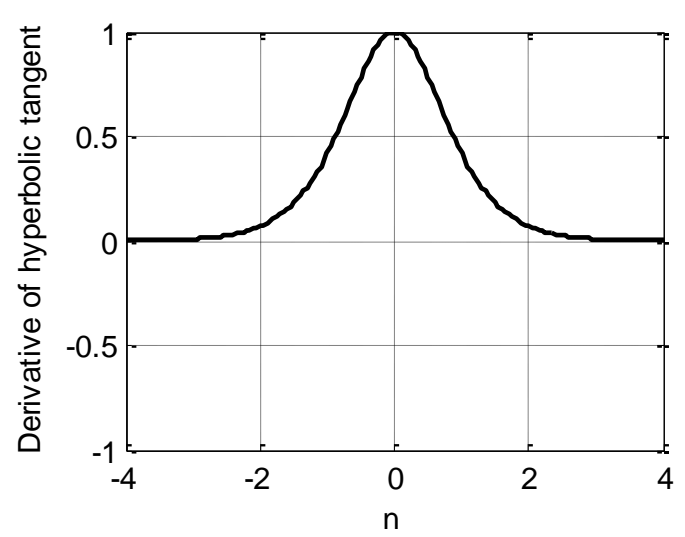

(b)

Figure 8: (a) Hyperbolic tangent function, (b) Its derivative.

\section{NEURAL NETWORK APPROXIMATION RESULTS}

We train the three NNs with the input and output data of Tables 1, 2, 3 (last two columns) and then each $\mathrm{NN}$ is asked to produce the full curves for each bed joint angle, for a set of 64 points. The results are shown in the figures below. 


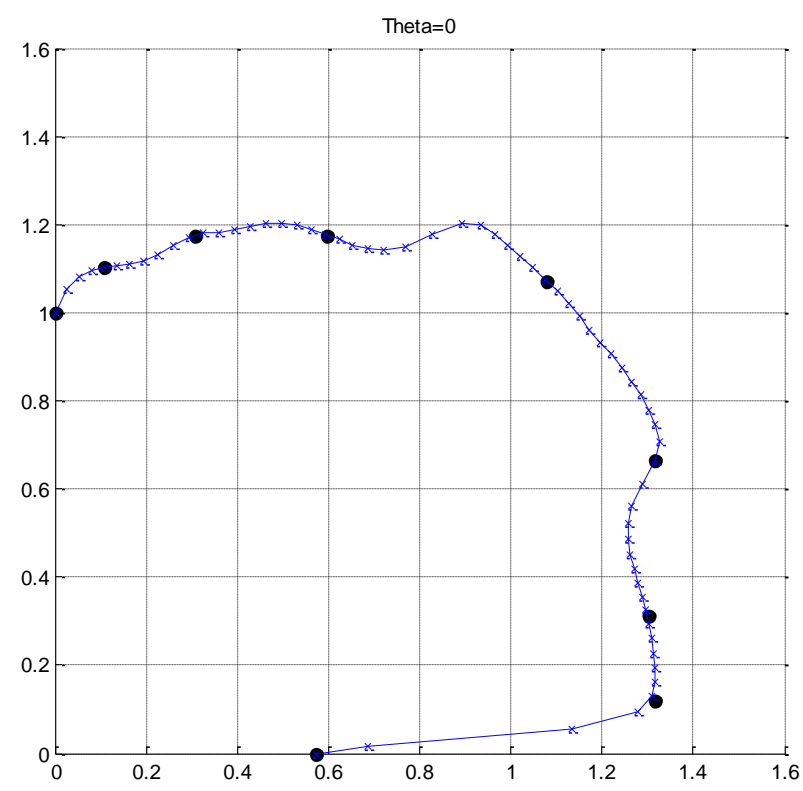

Figure 9: $\mathrm{NN}$ approximation result for the case $\theta=0^{\circ}$.

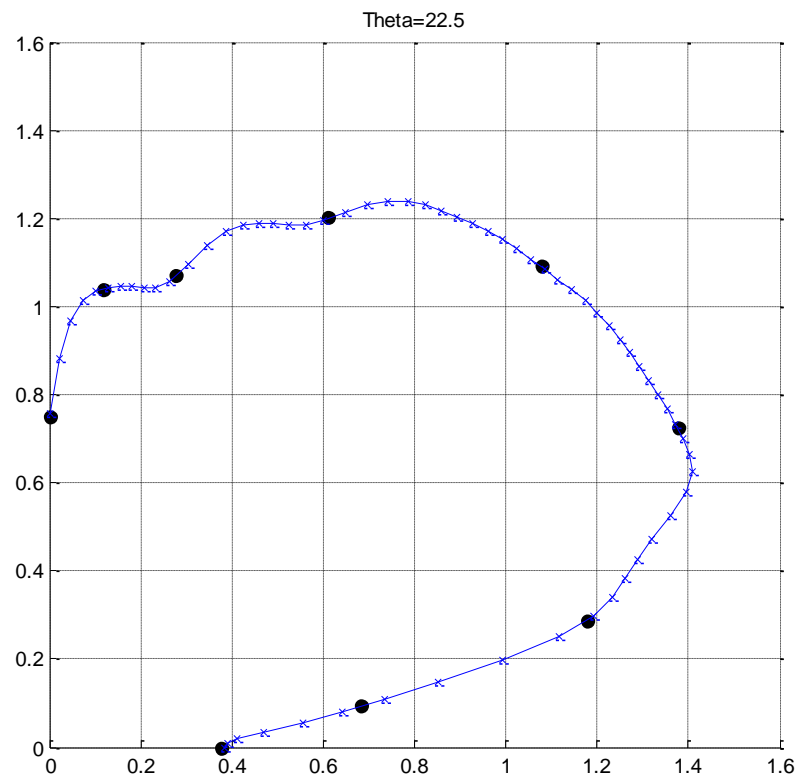

Figure 10: NN approximation result for the case $\theta=22.5^{\circ}$. 


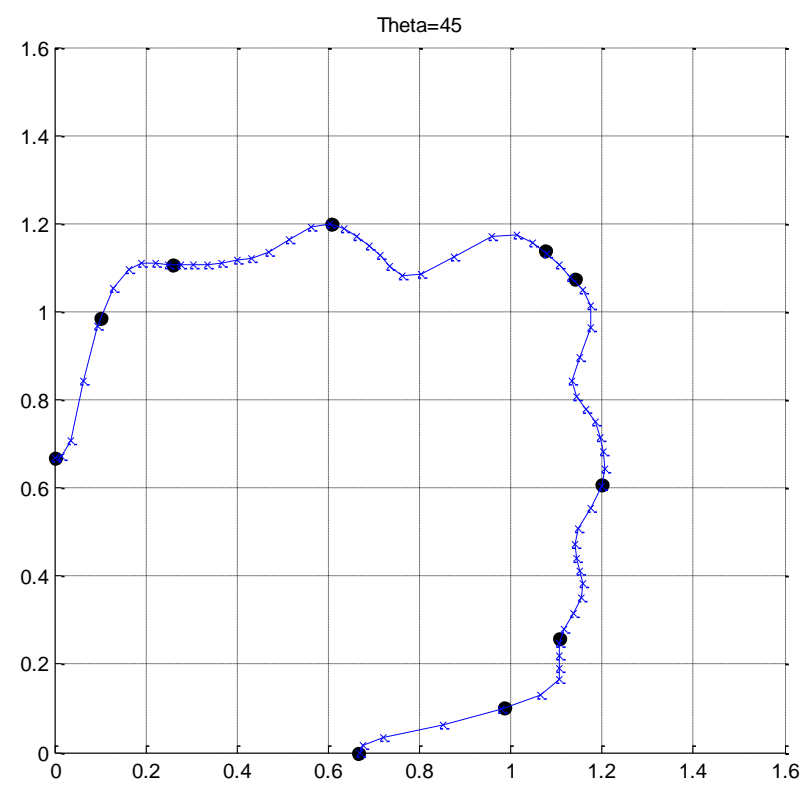

Figure 11: NN approximation result for the case $\theta=45^{\circ}$.

In the above figures, the black dots denote the input data, i.e. the data corresponding to the last two columns of Tables 1, 2, 3. The blue curve denotes the NN prediction of the fitting curve.

We can see that the $\mathrm{NN}$ manages to fit all the training data with excellent accuracy, while the approximation between the training data points appears to be adequate. An important characteristic is that for the third case $\left(\theta=45^{\circ}\right)$ the $\mathrm{NN}$ manages to produce results that are symmetric (with good accuracy) to the line of $45^{\circ}\left(\sigma_{\mathrm{I}}=\sigma_{\mathrm{II}}\right)$, an expected outcome due to the symmetry that masonry exhibits with respect to the $45^{\circ}$ degrees axis.

\section{CONCLUSIONS}

In the present study, preliminary results of an ongoing research project, on the failure of anisotropic brittle material such as masonry are presented. In particular, Neural Networks are used in order to approximate the experimental results for masonry failure [1]. For each angle $\theta$ a $\mathrm{NN}$ is trained with the experimental data as inputs and then each $\mathrm{NN}$ is asked to produce the whole failure curve for each angle as its output, filling also the gaps between the experimental points.

The NNs showed great performance in fitting the experimental input data, while they manages to fit all the training data with very good accuracy, also producing results that are symmetric (with good accuracy) to the line of $45^{\circ}\left(\sigma_{\mathrm{I}}=\sigma_{\mathrm{II}}\right)$ for the third case $\left(\theta=45^{\circ}\right)$. This is in very good agreement to the characteristic symmetry of the masonry. The results show the great potential of using $\mathrm{NN}$ for the approximation of the masonry failure under biaxial compressive stress.

A more detailed investigation has to be made regarding the use of $\mathrm{NN}$ for the generation of the failure surface for any angle $\theta$. The next research step would be to try to combine the results of the three separate NNs into a single NN that would also take the angle $\theta$ as its input. The trained NN should be then able to produce the whole 3D failure surface for any angle $\theta$. 


\section{REFERENCES}

[1] Page, A. W., "The biaxial compressive strength of brick masonry”, Proc. Instn Civ. Engrs, Part 2, Vol. 71, Sept., 893-906, 1981.

[2] Dhanasekar, M., Page, A. W., and Kleeman P. W., "The failure of brick masonry under biaxial stresses." Proceedings, The Institution of Civil Engineers, Part 2, 79, 295-313, 1985.

[3] Naraine, K., and Sinha, S., "Cyclic Behavior of Brick Masonry under Biaxial Compression." Journal of Structural Engineering, ASCE, Vol. 117, No. 5, 1336-1355, 1991.

[4] Scarpas, A., "Non-local Plasticity Softening Model for Brittle Materials / Experimental Evidence / Analytical Modelling / Preliminary Results." Research Report, Laboratory of Reinforced Concrete, National Technical University of Athens, 1991.

[5] Syrmakezis, C. A., Chronopoulos, M. P., Sophocleous, A. A., and Asteris, P. G., "Structural analysis methodology for historical buildings." Proceedings of the Fourth International Conference on Structural Studies of Historical Buildings, STREMA 95, Vol. 1, 373-382, 1995

[6] Syrmakezis, C. A., Asteris, P. G., and Sophocleous, A. A., "Earthquake resistant design of masonry tower structures." Proceedings, Fifth International Conference on Structural Studies of Historical Buildings, STREMA 97, 25-27 June, San Sebastian, Spain, 377386,1997

[7] Asteris, P. G., "Unified Yield Surface for the Nonlinear Analysis of Brittle Anisotropic Materials", Nonlinear Sci. Lett. A, Vol.4, No.2, 46-56, 2013.

[8] Samarasinghe, W., "The in-plane failure of brickwork," PhD thesis, University of Edinburgh, 1980.

[9] Page, A. W., "A biaxial failure criterion for brick masonry in the tension-tension range." The International journal of Masonry Construction, Vol. 1, No. 1, 1980.

[10] Tassios, Th. P., and Vachliotis, Ch. (1989). "Failure of masonry under heterosemous biaxial stresses." Proc. Int. Conf. Conservation of Stone, Masonry-Diagnosis, Repair and Strengthening, Athens.

[11] Syrmakezis, C. A., Asteris, P. G., "Masonry Failure Criterion Under Biaxial Stress State", Journal of Materials in Civil Engineering, Vol. 13, No. 1, 2001.

[12] Zhang, Y., Zhou, G., Xiong, Y., and Rafiq, M. "Techniques for Predicting Cracking Pattern of Masonry Wallet Using Artificial Neural Networks and Cellular Automata." J. Comput. Civ. Eng., 24(2), 161-172, 2010.

[13] Ahmed El-Shafie, T. Abdelazim, Aboelmagd Noureldin. "Neural network modeling of time-dependent creep deformations in masonry structures", Neural Computing and Applications, 19(4), 583-594, 2010.

[14] Zhou, G., Pan, D., Xu, X., and Rafiq, Y., "Innovative ANN Technique for Predicting Failure/Cracking Load of Masonry Wall Panel under Lateral Load.” J. Comput. Civ. Eng., 24(4), 377-387, 2010.

[15] A. Mathew, B. Kumar, B. P. Sinha, R. F. Pedreschi, Analysis of Masonry Panel under Biaxial Bending Using ANNs and CBR, Journal of Computing in Civil Engineering, 13(3), 170-177, 1999. 
[16] Lettvin, J. Y., Maturana, H. R., McCulloch, W. S., and Pitts, W. H. "What the Frog's Eye Tells the Frog's Brain." Proceedings of the Institute of Radic Engineers, 47(11), 1940-1951, 1959 